\title{
Hypoxic treatment of human dual placental perfusion induces a preeclampsia-like inflammatory response
}

\author{
Arjun Jain ${ }^{1,2,3}$, Henning Schneider ${ }^{3}$, Eldar Aliyev ${ }^{1}$, Fatimah Soydemir ${ }^{4,5}$, Marc Baumann ${ }^{2,3}$, Daniel Surbek ${ }^{2,3}$, \\ Matthias Hediger ${ }^{1,2}$, Paul Brownbill ${ }^{4,5}$ and Christiane Albrecht ${ }^{1,2}$
}

Preeclampsia is a human pregnancy-specific disorder characterized by a placental pro-inflammatory response in combination with an imbalance of angiogenic factors and clinical symptoms, including hypertension and proteinuria. Insufficient uteroplacental oxygenation in preeclampsia due to impaired trophoblast invasion during placentation is believed to be responsible for many of the molecular events leading to the clinical manifestations of this disease. We investigated the use of hypoxic treatment of the dual placental perfusion system as a model for preeclampsia. A modified perfusion technique allowed us to achieve a mean soluble oxygen tension within the intervillous space (IVS) of 5-7\% for normoxia and $<3 \%$ for hypoxia (as a model for preeclampsia). We assayed for the levels of different inflammatory cytokines, oxidative stress markers, as well as other factors, such as endothelin (ET)-1 that are known to be implicated as part of the inflammatory response in preeclampsia. Our results show a significant increase under hypoxia in the levels of different inflammatory cytokines, including IL-6 $(P=0.002)$, IL-8 $(P<0.0001)$, TNF- $\alpha(P=0.032)$ and IFN- $\gamma(P=0.009)$ at $360 \mathrm{~min}$ in maternal venous samples $(n=6)$. There was also a significant increase in ET-1 levels under hypoxia both on the maternal side at $30 \mathrm{~min}(P=0.003)$ and fetal side at $360 \mathrm{~min}(P=0.036)(n=6)$. Other markers of oxidative stress, including malondialdehyde and 8-iso-protaglandin $\mathrm{F} 2 \alpha(P=0.009)$ also show increased levels. Overall, these findings indicate that exposure of ex vivo dually perfused placental tissue to hypoxia provides a useful model for mimicking the inflammatory response characteristic of preeclampsia. This would therefore provide a powerful tool for studying and further delineating the molecular mechanisms involved in the underlying pathophysiology of preeclampsia.

Laboratory Investigation (2014) 94, 873-880; doi:10.1038/labinvest.2014.76; published online 16 June 2014

The ex vivo dual perfusion of the human placental cotyledon has proven a powerful tool to investigate a wide range of functions of this organ. ${ }^{1-3}$ This technique allows retention of a high level of structural organization of placental tissue, which better approximates to the in vivo state than other experimental systems using isolated tissues, cells or vessels. ${ }^{2}$ In addition to being used to study normal physiological functions of the placenta, this technique is also applied to explore pathological activity in the placenta, which can contribute to pregnancy disorders, such as preeclampsia. ${ }^{4}$ Preeclampsia is a human pregnancy-specific disorder that adversely affects maternal endothelial function and fetal intrauterine growth. ${ }^{5}$ One of the primary events in the pathophysiology of preeclampsia is deficient trophoblast invasion and remodeling of the maternal spiral arteries in the uterus during placentation.

The placental bed, the area of the uterus underlying the placenta, has a key role in supporting placental function by supplying oxygenated blood to the intervillous space (IVS). ${ }^{6}$ In the second and third trimesters of pregnancy, the placenta requires increasing access to the maternal blood supply. This is achieved by extensive remodeling of the maternal spiral arteries, which are the end arteries of the uteroplacental circulation that deliver blood directly into the placental IVS. ${ }^{7}$ Remodeling depends on one of the subtypes of the trophoblasts, which differentiates into tumor-like cells (extravillous cytotrophoblasts) that invade the lining of the pregnant uterus from 6 to 18 weeks of gestation. ${ }^{8}$ From 12 weeks on, the trophoblasts penetrate the maternal decidual

\footnotetext{
${ }^{1}$ Faculty of Medicine, Institute of Biochemistry and Molecular Medicine, University of Bern, Bern, Switzerland; ${ }^{2}$ Swiss National Center of Competence in Research, NCCR TransCure, University of Bern, Bern, Switzerland; ${ }^{3}$ Department of Obstetrics and Gynecology, University Hospital, University of Bern, Bern, Switzerland; ${ }^{4}$ Maternal and Fetal Health Research Centre, Faculty of Human and Medical Science, The University of Manchester, Manchester, UK and ${ }^{5}$ St Mary's Hospital, Central Manchester University Hospitals NHS Foundation Trust, Manchester Academic Health Science Centre, Manchester, UK

Correspondence: Professor C Albrecht, PhD, Faculty of Medicine, Institute of Biochemistry and Molecular Medicine, University of Bern Bühlstr. 28, Bern CH-3012, Switzerland.

E-mail: christiane.albrecht@ibmm.unibe.ch

Received 23 January 2014; accepted 15 April 2014
} 
tissue and extensively remodel the spiral arteries, including their distal myometrial segments, such that they lose their smooth muscle and become greatly dilated. ${ }^{7}$ This process completes by around 20 weeks, such that the maternal circulation can supply the expanding IVS of the placenta.

Reduced trophoblast invasion, resulting in deficient conversion of the uterine spiral arteries, is widely accepted as a key feature in the pathophysiology of preeclampsia. Deficient spiral artery conversion is associated with reduced placental perfusion. Reduced perfusion in the preeclamptic placenta gives rise to hypoxia, which has been linked to inflammatory processes, oxidative stress and even infarctions. ${ }^{9,10}$ Collectively, these give rise to increased production of pro-inflammatory cytokines and products of oxidative stress. Hypoxia is also a stimulus of endothelin (ET)-1, ${ }^{11}$ which is actually found to be elevated in preeclampsia compared with normal pregnancy and has been proposed to be a key factor in the pathogenesis of preeclampsia. ${ }^{12-16}$

A previous study by Soydemir et all ${ }^{17}$ adapted the dual placental perfusion technique to produce soluble oxygen tensions in the IVS associated with normal and preeclamptic pregnancy. The setup of these experiments involved reducing the mean soluble oxygen tension in the maternal-side IVS perfusate to $5-7 \%$ for normoxia, representative of physiological pregnancy, and to $<3 \%$ for hypoxia, which was used as a model for preeclampsia. ${ }^{17}$ Tissue viability remained unaffected in both the groups. Various parameters of energy metabolism as well as hypoxia-sensitive markers gave significant differences between groups, and it was concluded that this adaptation was suited to study different aspects of placental metabolism in relation to normal oxygenation and hypoxia-associated pregnancy diseases, such as preeclampsia. In the present study, we characterize the implications of low oxygen tension in the dual placental perfusion model with regards to the induction of proinflammatory responses and oxidative stress, which are associated with preeclampsia. This will allow to firstly verify the use of hypoxic treatment of the dual placental perfusion system as an ex vivo model for studying preeclampsia. This is important because this technique could provide a useful tool to dissect and further delineate the inflammatory pathways implicated under a controlled environment but still using 'live' human placental tissue that would be a close representation of the in vivo state. Secondly, this study would also highlight the importance of optimizing oxygen supply in the dual placental perfusion technique to approach in vivo conditions. As explained above, insufficient oxygen delivery to the placental tissue can stimulate oxidative stress, ${ }^{18-20}$ and fluctuations in oxygen levels would also create an ischemia/reperfusion-type insult that is an inducer of both oxidative stress and endoplasmic reticulum (ER) stress. ${ }^{13,21}$ Induction of these stress pathways is associated with the pathophysiology of preeclampsia ${ }^{22-24}$ and would have important implications for a number of studies using the dual placental perfusion technique.
Accordingly, we have measured the levels of different factors implicated in the pathophysiology of preeclampsia, including different cytokines, oxidative stress markers and ET-1, in order to characterize the pathways induced by hypoxia in the dual placental perfusion system and compared how well these correlate with previously published data on the in vivo levels of these factors in preeclampsia.

\section{MATERIALS AND METHODS \\ Dual Placental Perfusion}

The method of perfusion was carried out as previously described by Schneider et al ${ }^{1}$ and modified by Soydemir et al $^{17}$ In brief, human placentas were obtained from normal term pregnancies from vaginal deliveries or elective Caesarian sections. Informed written consent had been obtained from the patients before delivery, and the study had been approved by the local research Ethics Committee. An intact peripheral lobule was selected, and an associated branch of both the umbilical artery and vein was cannulated at the chorionic plate to establish the fetal circulation. On the maternal side of the placenta, the IVS was perfused by cannulae piercing the basal plate. Differently from most other studies, where not more than 3-5 maternal cannulae are used, 22 cannulae emanating from a bespoke perfusate distributor were inserted at alternating depths of 1 and $2 \mathrm{~cm}$ in order to improve the distribution of perfusate flow inside the IVS. For normoxia, maternal and fetal reservoirs were gassed with 15\% $\mathrm{O}_{2} /$ $5 \% \mathrm{CO}_{2} /$ balanced with $\mathrm{N}_{2}$ and $5 \% \mathrm{CO}_{2}$ /balanced with $\mathrm{N}_{2}$, respectively. For hypoxia, maternal and fetal reservoirs were gassed with $6 \% \mathrm{O}_{2} / 5 \% \mathrm{CO}_{2} /$ balanced with $\mathrm{N}_{2}$ and $5 \% \mathrm{CO}_{2} /$ balanced with $\mathrm{N}_{2}$, respectively. The setup of these experiments aimed for a median soluble oxygen tension in the IVS perfusate of $5-7 \%$ for normoxia, representative of physiological pregnancy, and of $<3 \%$ for hypoxia, which was used as a model for preeclampsia. ${ }^{17}$ These target values were achieved in a preliminary set of perfusions in eight out of nine lobules for normoxia and in all nine lobules for hypoxia. For normoxia, the median value of soluble oxygen tension was $4.9 \%$ (with a 25 th percentile at $2.5 \%$ and the 75 th percentile at $8.2 \%)$. In the hypoxic group, the median value was $1.2 \%$ (with a 25th percentile at $0.5 \%$ and the 75 th percentile at $4.0 \%)$. The difference of the median values in the two groups was highly significant. Further details of perfusate delivery and composition are described in our previous publication. ${ }^{17}$

Maternal and fetal venous perfusate samples had been obtained in the study of Soydemir et $\mathrm{al}^{17}$ and were collected under hypoxia or normoxia ( $n=8$ in each group) at the time points of 30, 180 and $360 \mathrm{~min}$. Samples were collected for $60 \mathrm{~s}$ into a Falcon tube (polypropylene) held on ice and centrifuged at $1500 \mathrm{~g}$ for $10 \mathrm{~min}$ at $4{ }^{\circ} \mathrm{C}$ to remove residual eluted blood.

\section{Multiplex Assay for Cytokine Measurements}

Maternal and fetal venous perfusate samples from both normoxia and hypoxia were analyzed for cytokine 
Table 1 Cytokine measurements in perfusate samples under normoxia and hypoxia

\begin{tabular}{|c|c|c|c|c|c|c|c|c|c|c|c|c|}
\hline \multirow[b]{2}{*}{ Target } & \multicolumn{3}{|c|}{$\begin{array}{c}\text { Maternal venous } \\
\text { samples under normoxia }(n=6)\end{array}$} & \multicolumn{3}{|c|}{$\begin{array}{c}\text { Maternal venous } \\
\text { samples under hypoxia }(n=6)\end{array}$} & \multicolumn{3}{|c|}{$\begin{array}{l}\text { Fetal venous samples } \\
\text { under normoxia }(n=6)\end{array}$} & \multicolumn{3}{|c|}{$\begin{array}{l}\text { Fetal venous samples } \\
\text { under hypoxia }(n=6)\end{array}$} \\
\hline & & & & & & & & & & & & \\
\hline cytokine & $30 \mathrm{~min}$ & $180 \mathrm{~min}$ & $360 \mathrm{~min}$ & $30 \mathrm{~min}$ & $180 \mathrm{~min}$ & $360 \mathrm{~min}$ & $30 \mathrm{~min}$ & $180 \mathrm{~min}$ & $360 \mathrm{~min}$ & $30 \mathrm{~min}$ & $180 \mathrm{~min}$ & $360 \mathrm{~min}$ \\
\hline IL-2 & $2.5 \pm 0.4$ & $5.6 \pm 0.8$ & $14.8 \pm 2.4$ & $0.8 \pm 0.4$ & $4.3 \pm 1.0$ & $13.0 \pm 1.8$ & $0.7 \pm 0.3$ & $2.0 \pm 0.4$ & $2.4 \pm 0.3$ & $1.00 \pm 0.4$ & $1.0 \pm 0.4$ & $2.7 \pm 0.7$ \\
\hline IL-4 & $1.3 \pm 0.1$ & $1.8 \pm 0.2$ & $2.8 \pm 0.2$ & $0.7 \pm 0.1$ & $1.5 \pm 0.1$ & $3.2 \pm 0.4$ & $0.7 \pm 0.2$ & $0.9 \pm 0.1$ & $1.0 \pm 0.1$ & $0.7 \pm 0.1$ & $0.8 \pm 0.1$ & $1.0 \pm 0.1$ \\
\hline IL-6 & $30.4 \pm 6.5$ & $886.3 \pm 139.5$ & $5781.6 \pm 420.4$ & $60.0 \pm 28.3$ & $698.6 \pm 108.4$ & $7758.5^{a} \pm 908.0$ & $6.2 \pm 1.7$ & $246.4 \pm 49.7$ & $1000.0 \pm 123.5$ & $4.5 \pm 1.8$ & $220.0 \pm 46.6$ & $456.8 \pm 50.9$ \\
\hline IL-8 & $31.5 \pm 4.6 \varepsilon$ & $8240.9 \pm 1473.7$ & $12064.6 \pm 1564.4$ & $29.2 \pm 7.6$ & $6423.8 \pm 1276.9$ & $27889.6^{a} \pm 6154.8$ & $3.9 \pm 0.8$ & $1596.1 \pm 466.0$ & $4529.8 \pm 560.4$ & $7.2 \pm 3.4$ & $1199.2 \pm 317.3$ & $1684.0 \pm 228.8$ \\
\hline IL-10 & $31.0 \pm 3.3$ & $42.9 \pm 4.1$ & $52.7 \pm 10.5$ & $18.4 \pm 6.2$ & $26.9 \pm 5.2$ & $34.3 \pm 9.5$ & $15.9 \pm 1.3$ & $19.7 \pm 2.1$ & $14.8 \pm 1.8$ & $11.9 \pm 3.5$ & $8.7^{a} \pm 1.7$ & $10.6 \pm 1.9$ \\
\hline $\mathrm{IFN}-\gamma$ & $37.9 \pm 4.5$ & $209.7 \pm 15.1$ & $539.9 \pm 20.7$ & $81.0 \pm 25.6$ & $234.1 \pm 21.5$ & $620.7^{\mathrm{a}} \pm 16.8$ & $35.5 \pm 7.5$ & $78.5 \pm 6.6$ & $112.4 \pm 18.9$ & $33.4 \pm 4.4$ & $72.4 \pm 15.7$ & $109.8 \pm 16.0$ \\
\hline TNF- $\alpha$ & $9.1 \pm 1.6$ & $191.5 \pm 66.2$ & $696.4 \pm 90.3$ & $6.7 \pm 1.0$ & $339.7 \pm 66.7$ & $908.7^{\mathrm{a}} \pm 92.8$ & $9.4 \pm 1.9$ & $35.6 \pm 12.9$ & $57.9 \pm 3.4$ & $4.7 \pm 0.6$ & $38.5 \pm 7.3$ & $55.4 \pm 17.9$ \\
\hline
\end{tabular}

Abbreviations: IFN, interferon; IL, interleukin; TNF, tumor necrosis factor.

Multiplex assay used to measure levels $(\mathrm{pg} / \mathrm{ml}$ ) of target cytokines in venous perfusate samples collected from normoxia or hypoxia at the indicated time points. Values in bold highlight samples that are significantly higher in hypoxia versus normoxia of samples collected at the same time point.

Statistical analysis was performed by one-way ANOVA followed by Fisher's PLSD multiple comparison test. Data are given as mean \pm s.e.m. $(n=6)$.

a Denotes a significant difference $(P<0.05)$ between venous perfusate samples at hypoxia vs normoxia collected from the same time point.

production (IL-2, IL-4, IL-6, IL-8, IL-10, TNF- $\alpha$, IFN- $\gamma$ ) using a Bio-Plex Human-cytokine kit (Bio-Rad Laboratories, Switzerland). Fifty microliters of each sample was analyzed on the Luminex 100 (Bio-Rad) according to the manufacturer's instructions.

\section{ELISA Measurements}

ET-1 was measured by a commercially available ELISA kit (Enzo Life Sciences, Switzerland) according to the manufacturer's instructions. The range of the standard curve was from 0.78 to $100 \mathrm{pg} / \mathrm{ml}$. Samples were assayed at $100 \mu \mathrm{l}$. The samples were read at $450 \mathrm{~nm}$ in a 96-well plate reader (Flex Station II, Bucher Biotec, Switzerland) using the Vmax4.8 software program.

Malondialdehyde (MDA) and 8-iso-protaglandin $\mathrm{F} 2 \alpha$ (8-isoprostane) were measured by commercially available ELISA kits from Cell Biolabs, Switzerland, according to the manufacturer's instructions. For MDA, the range of the standard curve was from 1.875 to $120 \mathrm{pmol} / \mathrm{mg}$. Samples were assayed at $100 \mu \mathrm{l}$. The samples were read at $450 \mathrm{~nm}$ in a 96-well plate reader (Flex Station II, Bucher Biotec) using the Vmax4.8 software program. For 8-isoprostane, the range of the standard curve was from 0.049 to $200 \mathrm{ng} / \mathrm{ml}$. Samples were assayed at $55 \mu \mathrm{l}$, after hydrolyzation with $2 \mathrm{M} \mathrm{NaOH}$, so as to measure the sum of free and esterified isoprostane. The samples were read at $450 \mathrm{~nm}$ in a 96-well plate reader (Flex Station II, Bucher Biotec) using the Vmax4.8 software program.

\section{Statistics}

The data were analyzed using a statistics package (StatView for Windows, version 5.0; SAS Institute, Cary, NC, USA). Statistical analysis was performed by analysis of variance (Fisher's Protected Least Significant Difference test) or nonparametric Mann-Whitney $U$-test, with $P<0.05$ being considered statistically significant. Data are presented as mean \pm s.e.m.

\section{RESULTS}

Induction of Pro-Inflammatory Cytokines under Hypoxia Key cytokines of both pro-inflammatory and anti-inflammatory pathways were measured at 30, 180 and $360 \mathrm{~min}$ in both maternal and fetal venous perfusate samples under hypoxia or normoxia (Table 1). Levels of the pro-inflammatory cytokines increased over time in both the maternal and fetal venous samples under normoxia or hypoxia. This culminated in significantly higher levels of IL-6, IL-8, TNF- $\alpha$ and IFN- $\gamma$ under hypoxia $v s$ normoxia in maternal venous samples at $360 \mathrm{~min}$ (Figure 1). It is also interesting to note the polarity of release of these cytokines, which was greater towards the maternal side of the placenta.

The levels of the anti-inflammatory cytokines were lower under hypoxia vs normoxia. This includes lower levels of IL-2, IL-4 and IL-10, although not significant, at each time point measured (Table 1). There was, however, a progressive increase in the levels of all cytokines over time.

\section{Induction of ET-1 under Hypoxia}

There were significantly higher ET-1 levels in the maternal venous samples under hypoxia $v s$ normoxia at $30 \mathrm{~min}$ (Figure 2a). Interestingly, there was a progressive decrease in ET-1 levels over time in the maternal venous perfusate samples under both normoxia and hypoxia. For the fetal venous samples, the converse was true. There was a progressive increase in ET-1 levels over time, culminating in significantly higher levels of ET-1 under hypoxia $v s$ normoxia at $360 \mathrm{~min}$ (Figure 2b). 
a

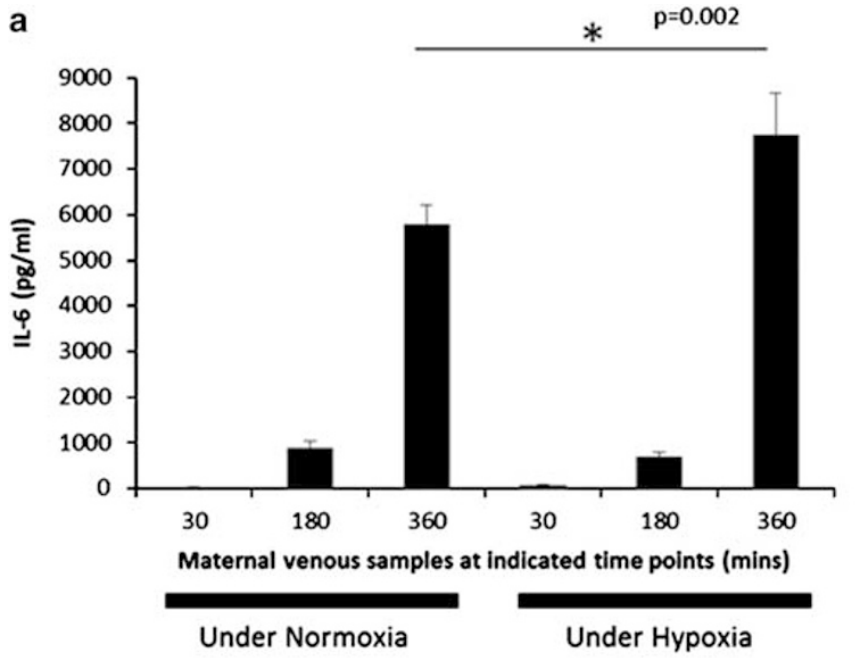

c

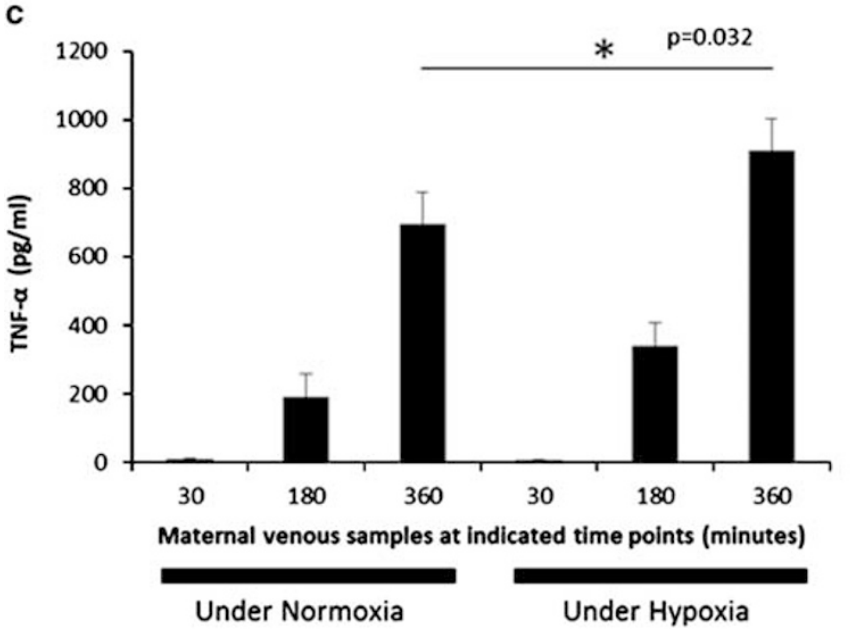

b

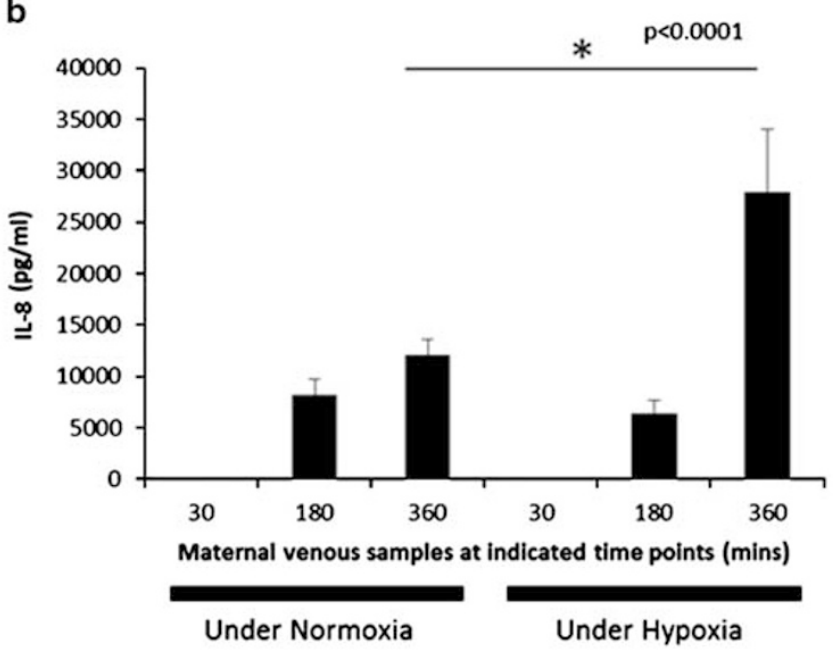

d

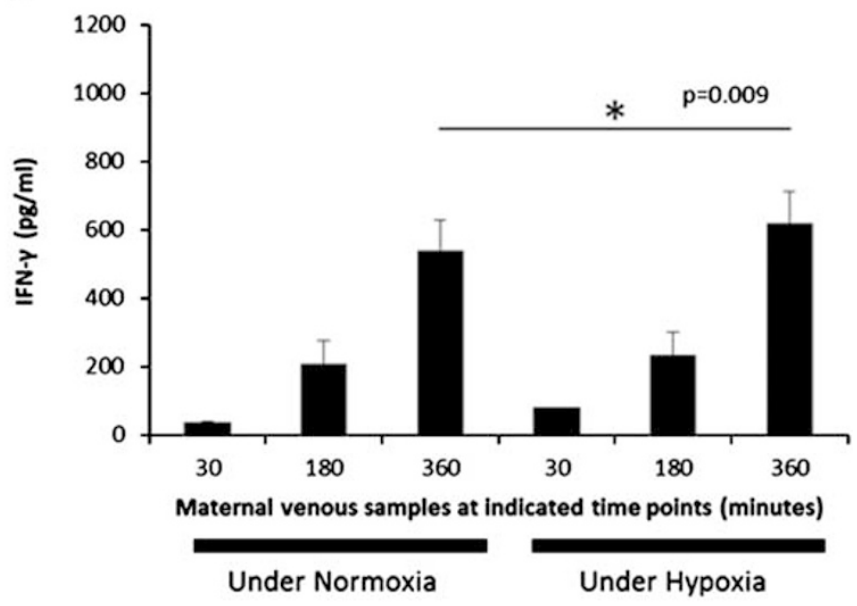

Figure 1 Pro-inflammatory cytokines stimulated under hypoxia vs normoxia include interleukin (IL)-6 (a), IL-8 (b), tumor necrosis factor (TNF)- $\alpha$ (c) and interferon (IFN)- $\gamma(\mathbf{d})$. There was a significant increase in target cytokine production under hypoxia vs normoxia in maternal venous samples collected at 360 min. Data are presented as mean \pm s.e.m. $(n=6)$.

Induction of Oxidative Stress Responses under Hypoxia Preeclampsia is characterized by increased lipid peroxidation and diminished antioxidant capacity. ${ }^{25}$ As clinical data showed increased plasma concentrations of MDA, a direct product of lipid peroxidation, in preeclamptic woman compared with normotensive controls, ${ }^{26}$ we measured the levels of MDA under hypoxic treatment of the dual placental perfusion system $v s$ normoxia. There were increased MDA levels under hypoxia $v s$ normoxia in maternal venous samples at $360 \mathrm{~min}$ (Figure 3a), which, however, did not reach statistical significance.

8-Isoprostane is another more sensitive marker of oxidative stress, which is induced under hypoxic conditions in preeclampsia. ${ }^{27,28}$ Significantly higher 8 -isoprostane levels were detected under hypoxia $v s$ normoxia in maternal venous perfusate samples at $360 \mathrm{~min}$ (Figure $3 \mathrm{~b}$ ).

\section{DISCUSSION}

Impaired placentation and consequent reduction in uterine blood flow exposes the placenta to hypoxia, and various studies have shown an increase in oxidative and ER stress, production of potent pro-inflammatory mediators and antiangiogenic factors in preeclampsia. ${ }^{29,30}$ In this study, we aimed to characterize whether ex vivo exposure of placental tissue to hypoxia using the dual perfusion system is suitable to study preeclampsia-associated responses. In order to use the dual perfusion model, median values of soluble oxygen tensions inside the IVS reflecting differences in oxygen content of media must be reasonably reproducible. As was recently shown by our group using direct measurement of oxygen tension inside the IVS, this requires a more elaborate maternal-side cannulation procedure than what is normally used. Satisfactory oxygenation was achieved by using 22 
a

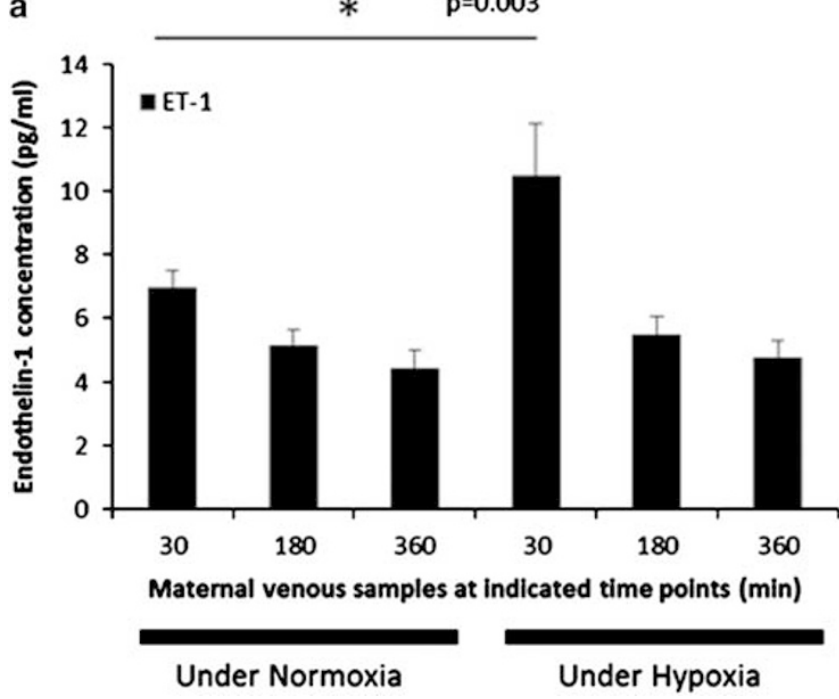

b

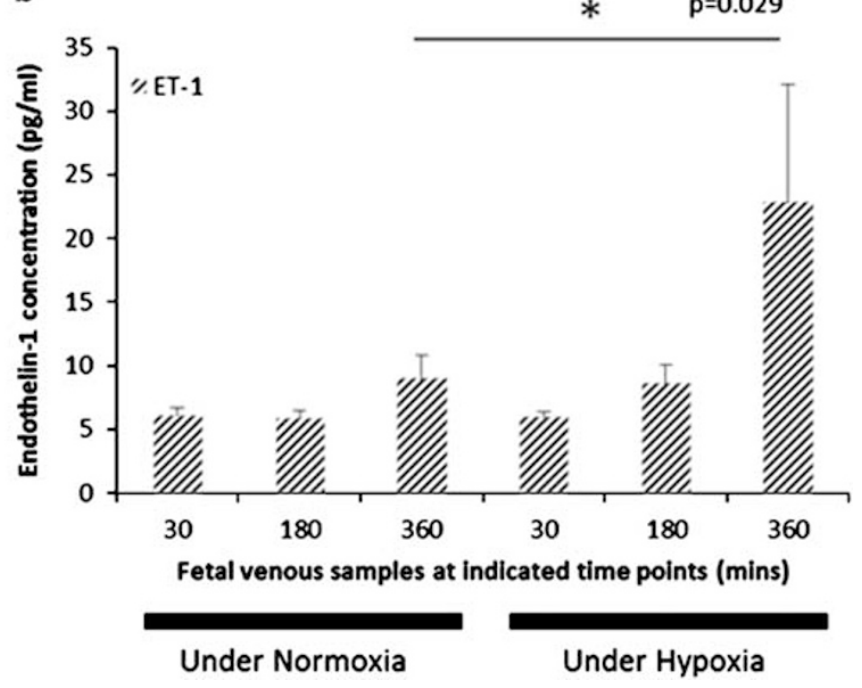

Figure 2 Endothelin (ET)-1 levels measured under hypoxia and normoxia in both maternal and fetal venous samples. There were significantly higher ET-1 levels under hypoxia vs normoxia in maternal venous samples collected at $30 \mathrm{~min}$ (a) and fetal venous samples at $360 \mathrm{~min}$ (b). Data are presented as mean \pm s.e.m. $(n=6)$.
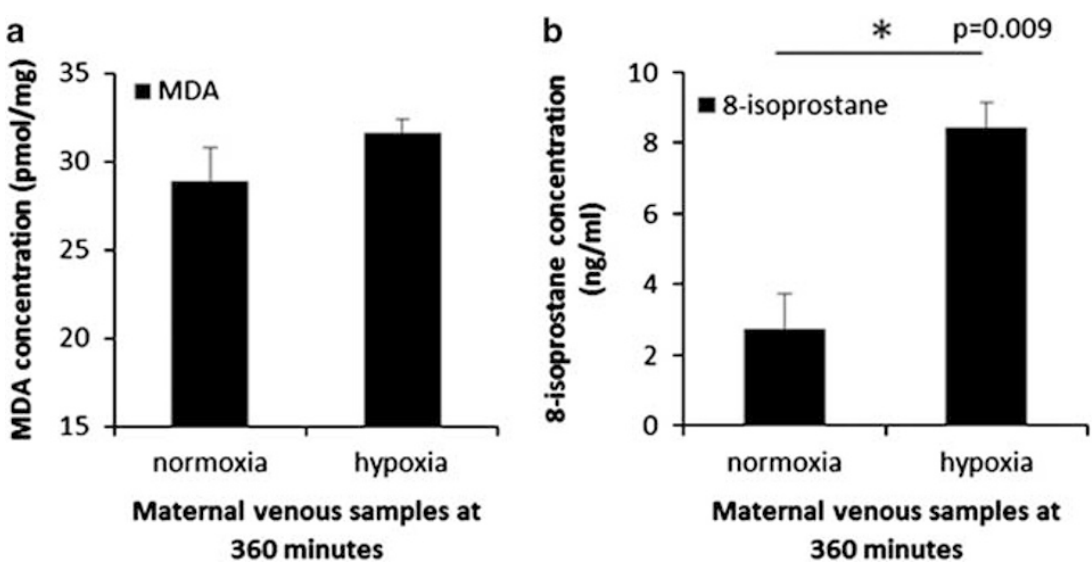

Figure 3 Products of lipid peroxidation, malondialdehyde (MDA) and 8-iso-protaglandin F2 $\alpha$ (8-isoprostane), are increased under hypoxia. MDA levels were higher under hypoxia compared with normoxia in maternal venous samples at $360 \mathrm{~min}$, but this increase was not significant (a). 8-Isoprostane levels were significantly higher under hypoxia vs normoxia in maternal venous samples at $360 \mathrm{~min}(\mathbf{b})$. Data are presented as mean \pm s.e.m. $(n=6)$.

maternal-side cannulae. ${ }^{17}$ In the current study, we show that hypoxia induces a significant increase in a number of proinflammatory cytokines that are known to be implicated in the pathophysiology of preeclampsia. There were significantly higher levels of TNF- $\alpha$, IFN- $\gamma$, IL-6 and IL-8 under hypoxia compared with normoxia.

An important feature of the systemic inflammatory response in preeclampsia is the shift in predominance from Th1-type to Th2-type immunity. In normal pregnancy, Th2type immunity prevails. Th1 cells synthesize TNF- $\alpha$ and IFN$\gamma$ among other factors. In preeclamptic patients, the decidual lymphocytes and peripheral blood mononuclear cells have been found to synthesize high levels of Th1 cytokines. ${ }^{31,32}$ Hence, our findings that hypoxic treatment of the dual placental perfusion system produces significantly higher levels of TNF- $\alpha$ and IFN- $\gamma$ closely parallel the in vivo response in preeclampsia. Furthermore, both IL-6 and IL-8 are found to be significantly increased in preeclampsia, ${ }^{33,34}$ and both these pro-inflammatory cytokines were also secreted at a significantly increased rate under hypoxic treatment of the dual placental perfusion system compared with the normoxic group. As there are very few maternal lymphatic cells present in this system, this response to hypoxia most likely originates from the placental trophoblast. The increase in pro-inflammatory cytokines would result in endothelial dysfunction or inappropriate endothelial cell activation, which are responsible for most of the common clinical manifestations of preeclampsia. ${ }^{35,36}$

Apart from the upregulation in pro-inflammatory cytokines, there is also an effect on the anti-inflammatory 
cytokines in preeclampsia compared with normotensive controls. Previous studies have reported reduced expression and release of IL-4 and IL-10 in preeclampsia compared with normotensive controls. ${ }^{37-39}$ Our data on the cytokine profile under hypoxic treatment of the dual placental perfusion are again consistent with this in vivo profile. Although not significant, we found a reduced level of both IL-4 and IL-10 under hypoxia $v s$ normoxia at each time point measured (data not shown). There was, however, a progressive increase in the levels of both cytokines over time, which might be representative of tissue infarct induced over time by ER stress (with induction of apoptosis and shedding of apoptotic debris from the syncytiotrophoblast) and/or oxidative stress.

In addition to cytokines, ET-1 has also been implicated in the inflammatory response of preeclampsia. In plasma from healthy pregnant women, the concentration of ET- 1 ranges from 5 to $10 \mathrm{pg} / \mathrm{ml}$, whereas it increases to $20-50 \mathrm{pg} / \mathrm{ml}$ in the presence of preeclampsia. ${ }^{16}$ Previous studies have shown a direct correlation between the levels of pro-inflammatory cytokines and ET-1 levels in preeclampsia. ${ }^{14,40,41}$ Hypoxia is a stimulus for ET-1 production in the placenta. ${ }^{11}$ However, following the significantly higher ET-1 levels under hypoxia $v s$ normoxia at $30 \mathrm{~min}$, there was a progressive reduction in ET-1 levels over time under both hypoxia and normoxia on the maternal side. ET- 1 levels are dependent on the rate of production as well as the rate of clearance. The reduction in maternal ET-1 levels over time might be reflective of increased clearance. ET-1 clearance is mediated via the ETB receptor. ${ }^{42}$ A study by Mondon et $a l^{43}$ found that the ETB receptor is expressed in term placental microvilli and suggested that the ETB receptor on the placental microvillous membrane may play a major role in ET-1 clearance by modulating the amounts of ET-1 in the maternal intervillous blood space. Given that the microvillus plasma membrane of the human placental syncytiotrophoblast is in direct contact with maternal blood (or the maternal perfusate in our model), this would be a source of ETB receptor-mediated ET1 clearance, which might explain the reduction in ET-1 levels over time. Under in vivo conditions, however, the placenta would be exposed to continual elevated ET-1 levels coming from the rest of the maternal circulation.

In the fetal compartment, ET-1 immunoreactivity has previously been observed in the endothelial lining of the umbilical vein and artery, as well as in the epithelium of the amniotic membrane. ${ }^{44}$ The same study also detected high levels of ET-1 in the fetal circulation and amniotic fluid. These data support a role of the placenta as a source of the increased ET-1 levels detected in the fetal circulation. In agreement with these findings, we found increasing levels of ET-1 in the fetal circuit under both normoxia and hypoxia. At $360 \mathrm{~min}$, there was significantly higher ET-1 in the hypoxic fetal venous samples compared with normoxia.

Previous studies have shown that ET-1 is a key mediator of both oxidative and ER stress, which are both part of and contribute to the inflammatory response in preeclampsia, ${ }^{13,16}$ and therefore the increased levels of ET-1 detected under hypoxic treatment of the dual placental perfusion further support the use of this system as a model for preeclampsia. Further studies should also explore the induction of ER stress markers in the perfused placental cotyledon under both hypoxia and normoxia, as this would give a more direct indication of any significant increase in ER stress responses concomitant to the increased ET-1 levels. ER stress is a multifaceted response, and previous work has shown that ET-1 induces expression of p-eIF2 $\alpha$, as well as the chaperone proteins GRP78 and GRP94, via the PERK and ATF6/IRE1 $\alpha$ pathways, respectively. Activation of these pathways can contribute to an inflammatory response through activation of nuclear factor-kappa B (NF- $\kappa \mathrm{B})$, a transcriptional regulator that has a key role in the induction of inflammation. ${ }^{45}$

In addition to factors directly implicated in the proinflammatory response in preeclampsia, there are several markers of oxidative stress that are detected in vivo. Inflammation and oxidative stress in preeclampsia are closely related. Oxidative stress in preeclampsia is not localized exclusively to the placenta but is dispersed throughout the maternal circulation ${ }^{46}$ and is an integral part of the systemic inflammatory response. The association of inflammation and oxidative stress is also supported by the finding that antioxidants, including antioxidant vitamins, which have a potential use in preventing preeclampsia, ${ }^{47}$ also have antiinflammatory actions. ${ }^{48}$ The markers of oxidative stress that have previously been shown to be elevated in preeclampsia include products of lipid peroxidation, such as MDA and 8isoprostane. A previous study from our group found an increased lipid profile in placentas from preeclamptic pregnancies compared with normotensive controls. ${ }^{49}$ The increased placental lipid profile in preeclampsia has been suggested to be indicative of placental pathology of this pregnancy disorder, which includes oxidative stress-induced lipid peroxide insult. Accordingly, several groups have found increased lipid peroxidation products in the plasma from women with preeclampsia compared with normotensive controls. ${ }^{26,50,51}$ In our study, although not significant, MDA levels showed a trend towards increased levels in maternal venous samples under hypoxia. A possible reason why there is no significant increase in MDA levels under hypoxia might be that the duration of the hypoxic treatment was too short. However, 8-isoprostane, a stable product of free radicalcatalyzed peroxidation of arachidonic acid in cell membrane phospholipids $^{52}$ which represents a more sensitive marker of lipid peroxidation, did give a significant increase under hypoxia $v s$ normoxia in maternal venous samples collected at $360 \mathrm{~min}$. Consistent with our findings, previous research indicates that this product of lipid peroxidation is significantly increased in woman with preeclampsia compared with normotensive controls. ${ }^{27,50,53,54}$ The study by Braekke et al ${ }^{28}$ found that the concentration of 8-isoprostane was much higher in plasma from the umbilical vein than artery, 
suggesting the placenta as a source of 8-isoprostane. Increased 8-isoprostane levels are postulated to cause endothelial cell dysfunction, ${ }^{53,55,56}$ which contributes to an inflammatory response.

Besides inflammatory processes, an imbalance of angiogenic factors has been proposed as a pathogenic factor in preeclampsia. ${ }^{57,58}$ The effect of normoxic $v s$ hypoxic treatment of placental dual perfusion on the steady-state release of proangiogenic factors, such as vascular endothelial growth factor (VEGF) and placental growth factor (PlGF), and the antiangiogenic factor soluble VEGFR-1 (sFlt-1) into the fetalside venous perfusate had been studied in this setting before, and no difference in the steady-state release of VEGF and sFlt1 into the fetal-side venous perfusate was found. However, PIGF release was significantly higher with hypoxic perfusion. ${ }^{17}$ This is in contrast to the decrease seen in plasma levels of PlGF in the second trimester of women, who later develop preeclampsia. ${ }^{59}$ This could possibly be explained by the nature of the placental tissue used in the perfusion experiments, namely from healthy and from term deliveries. It had been suggested that damage to the trophoblast leading to early onset preeclampsia occurs in the very early stages of embryonic development. ${ }^{60}$ Changes in plasma profile of proteins synthesized by the trophoblast found in early pregnancy have been recommended for the prediction of the development of the clinical picture of preeclampsia in the second half of pregnancy. ${ }^{61}$ The differences found for the angiogenesis-related factors were less obvious than for the cytokines related to inflammatory response found in this study. A possible explanation may be that dynamic changes in cytokine output are generally more of a short-term nature compared with the gene-based regulation of the synthesis of pro-angiogenic and antiangiogenic factors. The time required for gene upregulation and release of the product may exceed the 6 -h time course of the perfusion. ${ }^{62}$

\section{CONCLUSION}

In vitro methods mimicking the effects of reduced oxygen in the human placenta are important to understand the dysregulated molecular events characteristic of preeclampsia. As a model, the ex vivo dual perfusion system uniquely permits studies into the polarity of release for different hormones, cytokines and agonists, demonstrating the dynamic endocrine trafficking and secretion that exists within placental tissue. Our study indicates that hypoxic treatment of the dual placental perfusion system induces a preeclampsia-like inflammatory response, which would provide a useful model to further understand the underlying pathophysiology of preeclampsia.

Overall, our results indicate that under hypoxia the perfused placental cotyledon is under a pro-inflammatory state, with significantly higher levels ( $v s$ normoxia) of different factors detected at $360 \mathrm{~min}$. To further verify this system, it may be helpful to monitor the levels of the inflammatory and oxidative stress markers between the time points of 180 and
$360 \mathrm{~min}$, as significantly higher levels of these markers under hypoxia may be attained before the 6-h time point. In addition, previous work has demonstrated that hypoxia-reoxygenation is a more potent stimulus for oxidative stress than hypoxia alone. Deficient conversion of the maternal spiral arteries supplying the placenta does not just reduce oxygen supply giving placental ischemia but can also produce intermittent perfusion of the placenta inducing an ischemia-reperfusion-type insult that causes a more potent inflammatory response, triggering different oxidative and ER stress pathways. ${ }^{19,21,24}$ Therefore, it would be worthwhile to investigate the effect of hypoxiareoxygenation on the dual placental perfusion system, as this might give a stronger and more immediate response than that with hypoxic treatment alone.

This study also highlights the importance of optimizing oxygen delivery in the dual placental perfusion model. Hypoxia stimulated a preeclampsia-like inflammatory response in this system even in control experiments with healthy placentas, reflecting the need to optimize the perfusate supply and hence oxygen delivery to the perfused cotyledon. A full characterization of optimizing oxygen delivery to the perfused cotyledon in parallel with simultaneous measurements of oxidative and ER stress markers during the optimization procedure over time would allow to link (insufficient) oxygen delivery to the induction of oxidative and ER stress pathways, with potential implications for future research using the placenta perfusion model.

\section{DISCLOSURE/CONFLICT OF INTEREST}

The authors declare no conflict of interest.

1. Schneider $\mathrm{H}$, Panigel $\mathrm{M}$, Dancis J. Transfer across the perfused human placenta of antipyrine, sodium and leucine. Am J Obstet Gynecol 1972;114:822-828.

2. Dancis J. Why perfuse the human placenta. Contrib Gynecol Obstet 1985;13:1-4.

3. Leach L, Eaton BM, Westcott ED, et al. Effect of histamine on endothelial permeability and structure and adhesion molecules of the paracellular junctions of perfused human placental microvessels. Microvasc Res 1995;50:323-337.

4. Di Santo $S$, Sager R, Andres AC, et al. Dual in vitro perfusion of an isolated cotyledon as a model to study the implication of changes in the third trimester placenta on preeclampsia. Placenta 2007;28(Suppl A):S23-S32.

5. Hubel CA. Oxidative stress in the pathogenesis of preeclampsia. Proc Soc Exp Biol Med 1999;222:222-235.

6. Lyall F. Priming and remodelling of human placental bed spiral arteries during pregnancy-a review. Placenta 2005;26(Suppl A):S31-S36.

7. Pijnenborg $R$, Vercruysse $L$, Hanssens $M$. The uterine spiral arteries in human pregnancy: facts and controversies. Placenta 2006;27: 939-958.

8. Red-Horse K, Zhou Y, Genbacev O, et al. Trophoblast differentiation during embryo implantation and formation of the maternal-fetal interface. J Clin Invest 2004;114:744-754.

9. Redman CW, Sargent IL. Placental debris, oxidative stress and preeclampsia. Placenta 2000;21:597-602.

10. Sargent IL, Germain SJ, Sacks GP, et al. Trophoblast deportation and the maternal inflammatory response in pre-eclampsia. J Reprod Immunol 2003;59:153-160.

11. Hieda HS, Gomez-Sanchez CE. Hypoxia increases endothelin release in bovine endothelial cells in culture, but epinephrine, norepinephrine, serotonin, histamine and angiotensin II do not. Life Sci 1990;47:247-251. 
12. Jain A. Endothelin-1: a key pathological factor in pre-eclampsia? Reprod Biomed Online 2012;25:443-449.

13. Jain A, Olovsson M, Burton GJ, et al. Endothelin-1 induces endoplasmic reticulum stress by activating the PLC-IP(3) pathway: implications for placental pathophysiology in preeclampsia. Am J Pathol 2012;180: 2309-2320.

14. George EM, Granger JP. Endothelin: key mediator of hypertension in preeclampsia. Am J Hypertens 2011;24:964-969.

15. Tam Tam KB, George E, Cockrell K, et al. Endothelin type A receptor antagonist attenuates placental ischemia-induced hypertension and uterine vascular resistance. Am J Obstet Gynecol 2011;204:e1-e4.

16. Fiore $\mathrm{G}$, Florio $\mathrm{P}$, Micheli $\mathrm{L}$, et al. Endothelin-1 triggers placenta oxidative stress pathways: putative role in preeclampsia. J Clin Endocrinol Metab 2005;90:4205-4210.

17. Soydemir F, Kuruvilla S, Brown M, et al. Adapting in vitro dual perfusion of the human placenta to soluble oxygen tensions associated with normal and pre-eclamptic pregnancy. Lab Invest 2011;91: 181-189.

18. Zamudio S, Kovalenko O, Vanderlelie J, et al. Chronic hypoxia in vivo reduces placental oxidative stress. Placenta 2007;28:846-853.

19. Hung $\mathrm{TH}$, Burton GJ. Hypoxia and reoxygenation: a possible mechanism for placental oxidative stress in preeclampsia. Taiwan J Obstet Gynecol 2006;45:189-200.

20. Hung TH, Skepper JN, Burton GJ. In vitro ischemia-reperfusion injury in term human placenta as a model for oxidative stress in pathological pregnancies. Am J Pathol 2001;159:1031-1043.

21. Hung TH, Skepper JN, Charnock-Jones DS, et al. Hypoxiareoxygenation: a potent inducer of apoptotic changes in the human placenta and possible etiological factor in preeclampsia. Circ Res 2002;90:1274-1281.

22. Roberts JM, Hubel CA. Is oxidative stress the link in the two-stage model of pre-eclampsia? Lancet 1999;354:788-789.

23. Burton GJ, Jauniaux E. Placental oxidative stress: from miscarriage to preeclampsia. J Soc Gynecol Investig 2004;11:342-352.

24. Yung HW, Korolchuk S, Tolkovsky AM, et al. Endoplasmic reticulum stress exacerbates ischemia-reperfusion-induced apoptosis through attenuation of Akt protein synthesis in human choriocarcinoma cells. FASEB J 2007;21:872-884.

25. Gupta S, Aziz N, Sekhon L, et al. Lipid peroxidation and antioxidant status in preeclampsia: a systematic review. Obstet Gynecol Surv 2009;64:750-759.

26. Wu JJ. Lipid peroxidation in preeclamptic and eclamptic pregnancies. Eur J Obstet Gynecol Reprod Biol 1996;64:51-54.

27. Sampson MJ, Gopaul N, Davies IR, et al. Plasma F2 isoprostanes: direct evidence of increased free radical damage during acute hyperglycemia in type 2 diabetes. Diabetes Care 2002;25:537-541.

28. Braekke K, Harsem NK, Staff AC. Oxidative stress and antioxidant status in fetal circulation in preeclampsia. Pediatr Res 2006;60:560-564.

29. Burton GJ, Woods AW, Jauniaux $E$, et al. Rheological and physiological consequences of conversion of the maternal spiral arteries for uteroplacental blood flow during human pregnancy. Placenta 2009; 30:473-482

30. Redman CW, Sargent IL. Immunology of pre-eclampsia. Am J Reprod Immunol 2010;63:534-543.

31. Saito S, Sakai M, Sasaki Y, et al. Quantitative analysis of peripheral blood Th0, Th1, Th2 and the Th1:Th2 cell ratio during normal human pregnancy and preeclampsia. Clin Exp Immunol 1999;117:550-555.

32. Ramma W, Ahmed A. Is inflammation the cause of pre-eclampsia? Biochem Soc Trans 2011;39:1619-1627.

33. Bayram M, Bostanci MS, Celtemena MB, et al. Maternal inflammatory response in severe preeclamptic and preeclamptic pregnancies. J Clin Gynecol Obstet 2012;2:40-45.

34. Lamarca B, Brewer J, Wallace K. IL-6-induced pathophysiology during pre-eclampsia: potential therapeutic role for magnesium sulfate? Int J Infereron Cytokine Mediator Res 2011;2011:59-64.

35. Shamshirsaz AA, Paidas M, Krikun G. Preeclampsia, hypoxia, thrombosis, and inflammation. J Pregnancy 2012;2012:374047.

36. Redman CW, Sacks GP, Sargent IL. Preeclampsia: an excessive maternal inflammatory response to pregnancy. Am J Obstet Gynecol 1999;180:499-506.
37. Makris A, Xu B, Yu B, et al. Placental deficiency of interleukin-10 (IL-10) in preeclampsia and its relationship to an IL10 promoter polymorphism. Placenta 2006;27:445-451.

38. Rein DT, Breidenbach M, Honscheid B, et al. Preeclamptic women are deficient of interleukin-10 as assessed by cytokine release of trophoblast cells in vitro. Cytokine 2003;23:119-125.

39. Chatterjee $\mathrm{P}$, Kopriva $\mathrm{SE}, \mathrm{Chiasson} \mathrm{VL}$, et al. Interleukin-4 deficiency induces mild preeclampsia in mice. J Hypertens 2013;31:1414-1423.

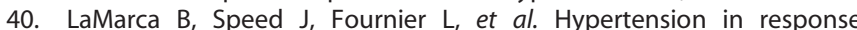
to chronic reductions in uterine perfusion in pregnant rats: effect of tumor necrosis factor-alpha blockade. Hypertension 2008;52: 1161-1167.

41. LaMarca BB, Cockrell K, Sullivan E, et al. Role of endothelin in mediating tumor necrosis factor-induced hypertension in pregnant rats. Hypertension 2005;46:82-86.

42. Fukuroda T, Fujikawa T, Ozaki $\mathrm{S}$, et al. Clearance of circulating endothelin- 1 by ETB receptors in rats. Biochem Biophys Res Commun 1994;199:1461-1465.

43. Mondon F, Anouar A, Ferre F. Endothelin receptor subtypes in the microvillous trophoblastic membrane of early gestation and term human placentas. Eur J Endocrinol 1998;139:231-237.

44. Hemsen A, Gillis C, Larsson $\mathrm{O}$, et al. Characterization, localization and actions of endothelins in umbilical vessels and placenta of man. Acta Physiol Scand 1991;143:395-404.

45. Rius J, Guma M, Schachtrup C, et al. NF-kappaB links innate immunity to the hypoxic response through transcriptional regulation of HIF1alpha. Nature 2008;453:807-811.

46. Hubel CA. Dyslipidemia, iron, and oxidative stress in preeclampsia: assessment of maternal and feto-placental interactions. Semin Reprod Endocrinol 1998;16:75-92.

47. Vadillo-Ortega F, Perichart-Perera $O$, Espino $S$, et al. Effect of supplementation during pregnancy with L-arginine and antioxidant vitamins in medical food on pre-eclampsia in high risk population: randomised controlled trial. BMJ 2011;342:d2901.

48. Hensley K, Robinson KA, Gabbita SP, et al. Reactive oxygen species, cell signaling, and cell injury. Free Radic Biol Med 2000;28:1456-1462.

49. Huang $X$, Jain A, Baumann $M$, et al. Increased placental phospholipid levels in pre-eclamptic pregnancies. Int J Mol Sci 2013;14:3487-3499.

50. Yanik FF, Amanvermez R, Yanik A, et al. Pre-eclampsia associated with increased lipid peroxidation and decreased serum vitamin $E$ levels. Int J Gynaecol Obstet 1999;64:27-33.

51. Barden A, Beilin LJ, Ritchie J, et al. Plasma and urinary 8-iso-prostane as an indicator of lipid peroxidation in pre-eclampsia and normal pregnancy. Clin Sci (Lond) 1996;91:711-718.

52. Roberts LJ, Morrow JD. Measurement of F-2-isoprostanes as an index of oxidative stress in vivo. Free Radic Biol Med 2000;28:505-513.

53. Walsh SW. Maternal-placental interactions of oxidative stress and antioxidants in preeclampsia. Semin Reprod Endocrinol 1998;16:93-104.

54. Walsh SW, Vaughan JE, Wang $\mathrm{Y}$, et al. Placental isoprostane is significantly increased in preeclampsia. FASEB J 2000;14:1289-1296.

55. Walsh SW, Vaughan JE, Wang $\mathrm{Y}$, et al. Placental isoprostane is significantly increased in preeclampsia. FASEB J 2000;14:1289-1296.

56. Mandang S, Manuelpillai U, Wallace EM. Oxidative stress increases placental and endothelial cell activin A secretion. J Endocrinol 2007; 192:485-493.

57. Ahmed A. Heparin-binding angiogenic growth factors in pregnancy. Placenta 1997;18:215-258.

58. Ramma W, Ahmed A. Is inflammation the cause of pre-eclampsia? Biochem Soc Trans 2011;39:1619-1627.

59. Levine RJ, Maynard SE, Qian C, et al. Circulating angiogenic factors and the risk of preeclampsia. N Engl J Med 2004;350:672-683.

60. Huppertz B. Placental origins of preeclampsia: challenging the current hypothesis. Hypertension 2008:51:970-975.

61. Huppertz B, Sammar M, Chefetz I, et al. Longitudinal determination of serum placental protein 13 during development of preeclampsia. Fetal Diagn Ther 2008;24:230-236.

62. Baumann MU, Bersinger NA, Mohaupt MG, et al. First-trimester serum levels of soluble endoglin and soluble fms-like tyrosine kinase- 1 as first-trimester markers for late-onset preeclampsia. Am J Obstet Gynecol 2008;199:e1-e6. 